\title{
MODELADO DEL ALUMNO EN JUEGOS EDUCATIVOS GESTIONADOS CON AGENTES SOFTWARE (JUEGAS) MULTIFUNCIONALES, EL CASO JuEGAS_ProVEAS
}

\author{
Yanilsa Milena Oyola Palomo, Yulieth Paola Argel Martínez, \\ Juan Carlos Giraldo Cardozo y Mónica Esther Castillo Gómez..
}

\begin{abstract}
RESUMEN
El presente artículo describe los criterios para estructurar el modelado del alumno en un "Video juego educativo gestionado con agentes software (JuEGAS)" multifuncional, usando como piloto el caso JuEGAS "Problemas de naturaleza verbal de estructura aditiva y sustractiva (ProVEAS)". El modelado del alumno se define como el estado actual de conocimiento que tiene el estudiante sobre un dominio temático.

El sistema multifuncional antes mencionado hace referencia a un software educativo que le brinda al docente la posibilidad de evaluar, ejercitar y tutorizar a los estudiantes en un dominio temático. En este caso, ese dominio corresponde a los problemas de naturaleza verbal de estructura aditiva y sustractiva y a las estrategias utilizadas por los niños entre 7 y 9 años para solucionar este tipo de problemas. En este orden de ideas, el modelado del alumno se creó con la posibilidad de permitir al software funcionar de manera dinámica en la medida en que se construye, por lo que se convierte en una estructura novedosa para el desarrollo de un JuEGAS ProVEAS multifuncional.
\end{abstract}

Para representar este modelo en el sistema se hace uso de la estructura de la red bayesiana y se diseña el "modelo unificado" como nueva propuesta en los diferentes tipos de modelados del alumno, existentes. Además, se definen ocho criterios indispensables a la hora de realizar el modelado del alumno con ayuda de la metodología "Software educativo, colaborativo, multimedia, adaptativo, lúdico e interactivo (secmali)" (Giraldo, 2007). La investigación utiliza el enfoque cualitativo, teniendo en cuenta un diseño exploratorio-explicativo y el método modelación que hace parte de la clasificación de los métodos de investigación y desarrollo de la informática definidos por (Barchini, 2005).

Palabras clave: videojuegos educativos, agentes pedagógicos inteligentes, problemas verbales de estructura aditiva y sustractiva, modelado del alumno, red bayesiana, sistema multifuncional.

30 Grupo de Investigación AVI: ambientes virtuales interactivos. Departamento de Informática Educativa, Universidad de Córdoba, Montería, Colombia. avi@unicordoba.edu.co. E-mails: aslinay05@gmail.com, licyuliethargel@hotmail.es, jgiraldocardozo@gmail.com,monicacg68@gmail.com. 


\begin{abstract}
This article describes the criteria for structuring Modeling Student in a MULTIFUNCTIONAL education game, using as JuEGAS_ProVEAS pilot case. The modeling of the student is defined as the current state of knowledge that the student have about a thematic domain.

The multifunctional system above refers to an educational software that gives teachers the opportunity to assess, to do exercises and bring tutoring, to students, about a thematic domain. In this case the domain corresponds to the problems of verbal nature of additive and subtractive structure, and strategies used by children between 7 and 9 years to solve such problems. In this vein, student modeling was created to allow the software can operate dynamically to the extent that is constructed, so that it becomes a novel structure for the development of a multifunctional JuEGAS_ProVEAS. To represent this model in the system, is makes use of Bayesian network structure and is designed the "unified model" as a new proposal addressing different types of student modeling that existed. In addition, it define 8 criteria necessary, for to desing the modeling student, when using the SECMALI methodology (Giraldo, 2007). The research uses the qualitative approach, taking into account a exploratory-explanatory design and modeling method that is part of the classification of research methods and development of information defined by (Barchini, 2005).
\end{abstract}

Key words: educational Games, Intelligent Pedagogical Agents, Word Problems additive and subtractive structure, JuEGAS, ProVEAS, Student modeling, Bayesian network, system MULTIFUNCTIONAL.

Recibido: 30 de abril de 2009

Aceptado: 5 de mayo de 2010

\title{
INTRODUCCIÓN
}

El problema surge ante la necesidad de sistematizar la creación del modelado del alumno para productos JuEGAS (Giraldo, 2007), usando como dominio de conocimiento las estrategias para la resolución de ProVEAS (Castillo, 2008). Puesto que en Castillo encontramos elementos clave para crear un software innovador que permite brindar al docente la posibilidad de indagar las dificultades que presentan sus estudiantes en la solución de "Problemas de naturaleza Verbal de Estructura Aditiva y Sustractiva (ProVEAS)", enseñarles el tipo de estrategia adecuada para solucionarlos, y ejercitarlos para fortalecer sus estrategias; de esta manera convertirlos en solucionadores exitosos de ProVEAS. 


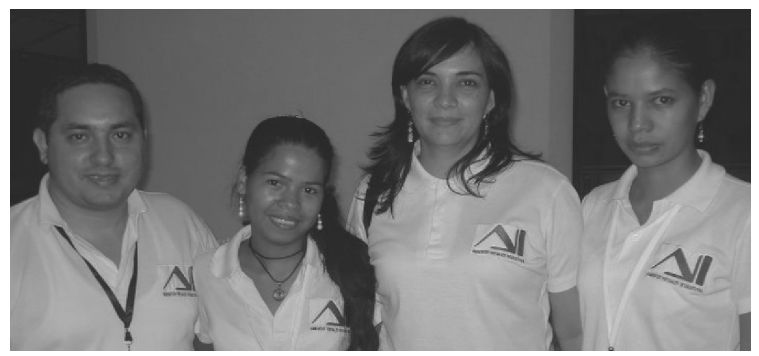

Fotografía 1. Responsables del Proyecto JuEGAS ProVEAS Multifuncional Modelado del Alumno

Además, (Giraldo, 2007) diseña la metodología "Software Educativo, Colaborativo, Multimedia, Adaptativo, Lúdico e Interactivo (SECMALI)" para guiar el proceso de construcción de los JuEGAS: Juegos educativos gestionados con agentes software, es decir, juegos con una clara intención pedagógica, soportados por un sistema tutor inteligente. Sin embargo, Giraldo en su metodología, no define los pasos a seguir en la construcción del modelado del alumno por lo que se hace pertinente llevar a cabo la investigación.

\section{ESTADO DE LA INFORMACIÓN}

\section{Modelado del estudiante}

El modelo del estudiante ha tomado diversas connotaciones. (Varlehn, 1998), lo define como: un modelo de simulación, este no describe la probabilidad de que un estudiante realice algo bien o no, describe los procesos por los cuales el estudiante recoge información sobre un problema y realiza aserciones. De la misma forma que un modelo de simulación de un circuito eléctrico puede predecir los estados internos de varios de sus componentes, por ejemplo el voltaje, un modelo de simulación de un estudiante puede predecir qué hará el estudiante próximamente.

(Vanlehn, Niu y Gertner, 1998) retoman el modelamiento del estudiante, afirmando que con este modelo se pueden hacer inferencias sobre el estado actual de un estudiante, considerando el tipo de conocimiento (procedimental, declarativo o cualitativo) y diferenciando entre el estudiante y el experto, puesto que existen elementos que el estudiante no tiene y el experto sí, así como otros que el estudiante tiene pero el experto no. Además, Varlehn al igual que (Millan, 2000) y (Cataldi, Salgueiro y Lage, 2006); afirman, que el modelado del alumno es el componente del "Sistema tutor inteligente (STI)" que representa el estado actual del conocimiento del alumno; el proceso que lo manipula, se llama diagnóstico. Ambos componentes deben diseñarse juntos, este problema de diseño es el que se conoce como el problema del modelado del alumno.

En este orden de ideas, (Holt, et al., 1994 c. p. Moreno 2002) plantea que: "el término modelo del estudiante, significa una representación de las creencias que el sistema tiene acerca del estudiante y es, por lo tanto, una representación abstracta del estudiante en el 
sistema". Para abordar el problema del modelado del alumno se desarrollaron principalmente tres paradigmas del manejo de la incertidumbre: la teoría de la evidencia de Dempster-Shafer (DST), la lógica Fuzzy y la red bayesiana. De las teorías antes mencionadas, se adopta la red bayesiana como la estructura para hacer evidente el modelado del alumno en el JuEGAS ProVEAS multifuncional, autores como (Millan 2000) y (Ramírez, 2005) hacen uso de la red bayesiana para explicar la incertidumbre en la creación del modelado del alumno.

\section{Problemas de naturaleza verbal de estructura aditiva y sustractiva (proveas)}

Teniendo en cuenta los ProVEAS, Riley, Cols, citados por Castillo, 2008, los definen como aquellos problemas que implican hacer su representación, es decir, poseer suficiente cantidad de datos y conceptos para luego encontrar la solución utilizando reglas de la aritmética o del álgebra. Además (Orton, citado por Castillo, 2008), considera que "la resolución de problemas se concibe como generadora de un proceso a través del cual quien aprende combina elementos del procedimiento, reglas, técnicas, destrezas y conceptos previamente adquiridos para dar soluciones a una situación nueva".

De igual forma (Riley, Greeno, Heller, citado por Castillo, 2008), clasifican los problemas verbales de suma y resta, según la estructura semántica en cuatro niveles de complejidad: cambio, combinación (llamada de reunión por ciertos autores), comparación e igualación. A su vez, cada nivel de estos está compuesto por 6 subniveles de complejidad.

Tabla 1. Clasificación de los PNV. (Riley, Greeno y Heller, 1983), tomado de (Castillo, 2008)

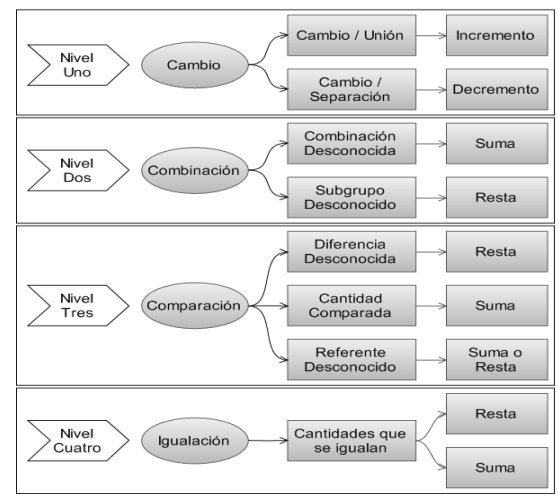

En síntesis, los problemas de naturaleza verbal implican hacer una representación mental para solucionar situaciones nuevas con la ayuda de reglas de la aritmética o del álgebra.

Para resolver los ProVEAS, los niños entre 7 y 9 años utilizan algunas estrategias para llegar a la solución de estos problemas. Los siguientes autores (Weinstein, citado en Castillo, 2008), define una estrategia como una serie de competencias que los investigadores han postulado como necesarias, o útiles, para el aprendizaje efectivo y la retención de la información y su uso posterior. 
En este orden de ideas, Derry y Murphy, citados en Castillo, 2008, señalan que una estrategia se refiere al conjunto de actividades mentales empleadas por el individuo en una situación particular de aprendizaje, para facilitar la adquisición de conocimiento. Para Gagné, citado en Castillo, 2008, la estrategia es una habilidad intelectual parcialmente entrenable, que se desarrolla como resultado de la experiencia y de la inteligencia. Dice también que las estrategias son procesos mentales superiores que se utilizan en función de una meta propuesta. En síntesis, una estrategia es una habilidad intelectual que un sujeto desarrolla al enfrentarse a una situación en particular.

(Castillo, 2008), tiene en cuenta tres tipos de estrategias para la solución de problemas de naturaleza verbal de estructura aditivas y sustractivas, dichas estrategias son planteadas por los siguientes autores: Kantowsky, Schoenfeld, Polya y Hegarty.

Estrategia método directo: al principio, los niños emplean modelos concretos que representan directamente su concepto informal de la resta o de la suma como "quitar" "agregar algo". Usando objetos o los dedos de la mano, se construye el conjunto mayor "a". Se quitan o se suman de él el conjunto menor "b". La respuesta es el número de los objetos que quedan u objetos que se agregan y que el niño cuenta una vez que ha hecho la separación.

Estrategia método hacia atrás: contar hacia atrás hasta un número dado. Se comienza la secuencia de conteo hacia atrás, comenzando desde número mayor "a", y se continúa tantos pasos como sean necesarios para llegar atrás, al número menor "b”. La respuesta es el número de palabras en la secuencia de conteo.

Estrategia método hacia delante: contar hacia adelante a partir de un número dado. Se comienza la secuencia de conteo hacia adelante empezando con el número menor "b" y se continúa hasta que se llega a "a". La respuesta es el número de palabras contadas en la secuencia.

\section{Juegos educativos gestionados con agentes software (juegas)}

Desde el punto de vista de la formación (Carro, Breda, Castillo, Bajuelos, 2002) argumentan que un juego educativo está formado por un conjunto de actividades que los usuarios deben realizar. Cada una de estas actividades tiene unos objetivos, que representan el conocimiento que los usuarios deben adquirir durante la realización de la misma. Una actividad puede tener asociados uno o varios juegos informáticos, juegos con los que los usuarios interactúan para adquirir los conocimientos o habilidades descritas en los objetivos de la actividad.

Carro y otros abordan también el tema de los juegos educativos adaptativos. Estos consisten en que el conjunto de actividades que pueden ser realizadas en cada momento, se seleccionan dinámicamente para cada usuario en particular, con el objetivo de facilitarle el aprendizaje. De igual forma, (Martínez, 2005), afirma que un juego adaptativo y colaborativo (juego AC) está formado por un conjunto de actividades que los participantes deberán realizar individualmente o de forma colaborativa. 
Según (Giraldo, 2007), “Los Juegos Educativos, colaborativos, adaptativos, Gestionados con Agentes Software (JuEGAS), son un tipo particular de producto educativo que aprovecha las ventajas que brinda la multimedia y se basa en el juego como formato de presentación. Aprovecha la tecnología de agentes para brindar adaptación al proceso de aprendizaje del usuario y una plataforma para el desarrollo de actividades de tipo colaborativo".

Un JuEGAS es entonces un software educativo estructurado como video juego: juegos de rol, micromundos lúdicos interactivos, mundos virtuales, etc., que tiene en cuenta el modelado del usuario y la posibilidad de asistencia adaptada al estudiante o usuario. La estrategia de trabajo colaborativo se incluye dentro de la actividad lúdica de forma natural, es decir, que solo pueden completarse las actividades con la participación significativa de otros (Giraldo, 2007).

Tabla 2. Resumen de las características de los JuEGA, adaptado de (GIRALDO, 2007)

\begin{tabular}{|c|c|c|}
\hline \multicolumn{3}{|c|}{ JuEGAS } \\
\hline Pedagógico & Comunicacional & Computacional \\
\hline $\begin{array}{l}\text { Los JuEGAS se convierten en: } \\
\text { Educativo porque poseen un modelo } \\
\text { pedagógico claroy definen estrategias } \\
\text { de enseñanza y aprendizaje que } \\
\text { sustentan el desarrollo de la historia } \\
\text { y los retos presentados. } \\
\text { Colaborativo porque posibilita el } \\
\text { trabajo en grupo entre los jugadores. } \\
\text { Adaptativo debido a que realizan } \\
\text { una asistencia al estudiante, en } \\
\text { función de su desempeño en las } \\
\text { tareas asignadas. }\end{array}$ & $\begin{array}{l}\text { Los JuEGAS son juegos debido a } \\
\text { que: } \\
\text {-Incorporan características de los } \\
\text { micromundos. } \\
\text {-Presentan un modelo simplificado } \\
\text { y significativo de un dominio. } \\
\text {-Son altamente interactivoyarticulado } \\
\text { en un contexto lúdico. } \\
\text {-Presenta retos que deben ser } \\
\text { sorteados conniveles decomplejidad } \\
\text { creciente. }\end{array}$ & $\begin{array}{l}\text { Los JuEGAS : } \\
\text {-Se apoya en las técnicas de ingeniería } \\
\text { de software orientado a agentes. } \\
\text {-Su arquitectura soporta técnicas } \\
\text { de trabajo en grupo que facilitan el } \\
\text { desarrollo de actividades de trabajo } \\
\text { colaborativo. } \\
\text {-Brinda adaptación al usuario con la } \\
\text { ayuda de los algoritmos basados en } \\
\text { inteligencia artificial. }\end{array}$ \\
\hline
\end{tabular}

Con base en lo anterior, se puede concluir que los juegos educativos gestionados con agentes software brindan elementos que facilitan el aprendizaje de forma lúdica, interactiva y colaborativa en el desarrollo de las actividades o tareas planteadas dentro del juego. Además, buscan adaptarse a las necesidades educativas del usuario.

\section{Sistema multifuncional}

El sistema multifuncional se plantea como un software dinámico e innovador en su desarrollo, creado con la finalidad de adaptarse a las necesidades de los estudiantes a través de la implementación de 3 ámbitos pedagógico como los son: evaluar, ejercitar y tutorizar las estrategias de aprendizaje de los estudiantes al momento de dar solución a un problema de naturaleza verbal de estructura aditiva y sustractiva planteado. 
Este software tendrá la finalidad de permitir, a través de un mismo sistema, ofrecer al estudiante una tutoría, presentar módulos de ejercitación y ofrecer la posibilidad de evaluar los avances de los estudiantes. Todo lo anterior controlado de manera sencilla por el docente y con la posibilidad de actualizar los contenidos sin alterar la lógica del programa.

De acuerdo con las revisiones bibliográficas realizadas, no se encontró información sobre el sistema multifuncional. Para explicar las características del sistema se consultaron autores que han abordado temáticas acerca de un sistema evaluador, de un sistema tutor y un sistema ejercitador que brindan elementos explicativos en la comprensión del sistema multifuncional.

Zambrano, (1989) hace un breve resumen sobre la educación apoyada por computador inteligentemente (EACI), define los Sistemas de Ejercitación y Practica (SEP) y nombra las características principales de este tipo de software educativo, como también enuncia algunos de los objetos estructurados utilizados en la representación del conocimiento (redes semánticas, marcos y sistemas orientados a objetos).

En esta misma línea (Ramírez, 2005), diseñó y construyó un prototipo de ejercitador de contabilidad. El sistema es capaz de generar, de forma aleatoria y automática, tres tipos de ejercicios (de demostración, guiados y de aplicación) en los que el estudiante puede ir asumiendo de forma gradual la autonomía, teniendo a su disposición información y ayuda (del entorno y adaptativa) para resolverlos.

Conjugando dos de los sistemas antes mencionados, (Carretero, 2001), aporta un módulo tutor-evaluador que supone una innovación en su concepción, pues plantea al alumno la resolución de ejercicios completos, y no cuestiones aisladas: "Este sistema tutor-evaluador es un paso hacia un futuro en el que la misión del profesor va a estar más centrada en aspectos como la motivación de los alumnos o el establecimiento del contenido de los programas, y no en la mera transmisión de conocimientos. El programa permitirá al estudiante aprender de una forma guiada y con mayor independencia del profesor, pudiéndose llegar en un futuro a que la evaluación formal de los alumnos se realice con una intervención reducida por parte del profesor".

\section{METODOLOGÍA}

Esta investigación comienza con un enfoque de tipo exploratorio, debido a que existen pocos antecedentes que expliquen el modelado del alumno en cuanto a su modelo teórico o a su aplicación práctica dentro de la metodología SECMALI y concluye con un enfoque Explicativo debido a que los estudios explicativos van más allá de la descripción de conceptos o fenómenos o del establecimiento de relaciones entre conceptos, su interés se centra en explicar por qué ocurre un fenómeno y en qué condiciones se da éste, o por qué dos o más variables están. Además, se enmarca desde el enfoque cualitativo. (Hernández, Fernández, Baptista, 1997). 


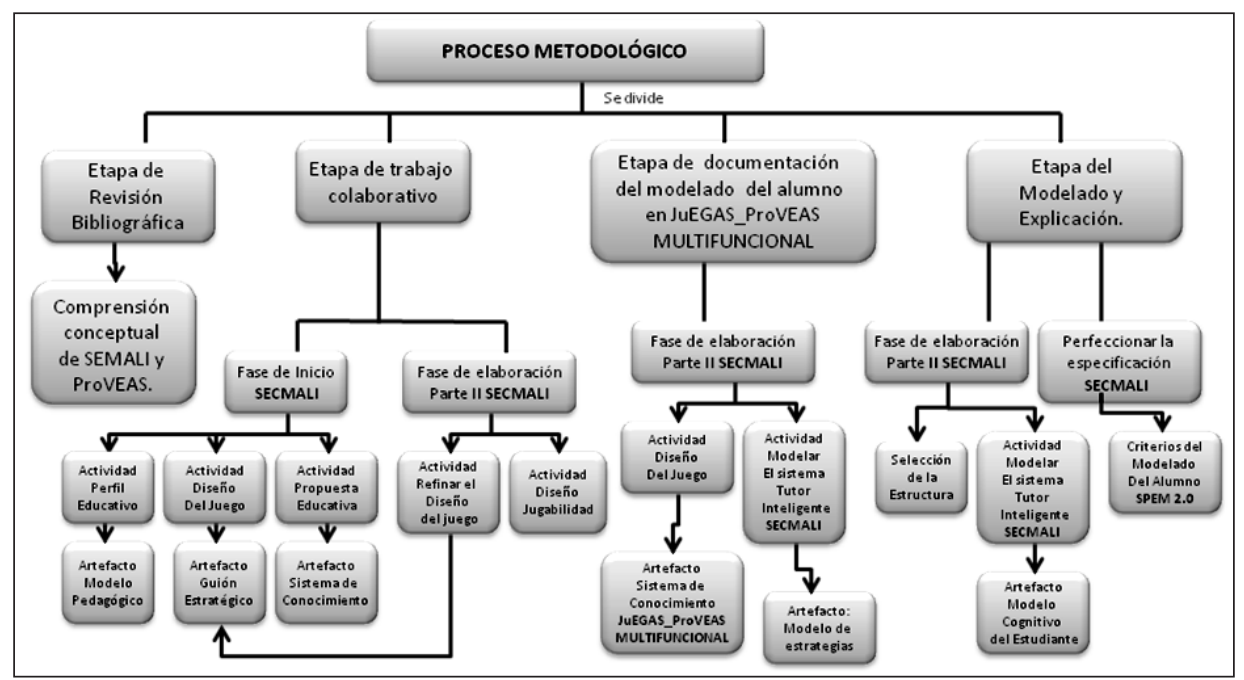

Figura 1. Etapas de la investigación

Método: (BARCHINI, 2005), según la clasificación de los métodos de investigación y desarrollo de la informática, esta investigación se enmarca dentro de los métodos lógicos en la categoría de Métodos Lógicos de soporte, específicamente en los métodos de modelación.

Métodos lógicos: se basan en la utilización del pensamiento en sus distintas funciones de deducción, análisis y síntesis para llegar al conocimiento.

Métodos lógicos de soporte: es la categoría de los métodos lógicos que contribuyen a la obtención de conocimiento.

Modelación: es una categoría de los métodos lógicos de soporte que promueve la creación de abstracciones con el propósito de explicar la realidad. El modelo es el sustituto del objeto de investigación.

Etapas de investigación: la investigación consta de cuatro etapas que se dividen a su vez en subetapas:

\section{Etapa de revisión bibliográfica}

Se realizó un análisis de la metodología SECMALi y del anteproyecto ProVEAS, con el fin de identificar las posibles problemáticas que podrían ser abordadas en la investigación y los elementos clave que servirían como punto de partida en el modelado del alumno. Para ello se hizo una revisión bibliografía bastante amplia de los temas principales a través de la búsqueda, selección, organización y representación de la información. Como resultado de la revisión bibliográfica se establecieron las categorías de investigación: JuEGAS, ProVEAS, Modelado del Alumno y Sistema Multifuncional. 


\section{Etapa de trabajo colaborativo}

En esta etapa se especificaron los procesos de la metodología SECMALI (roles, tareas, artefactos y actividades), que tienen mayor relación con la investigación y se desarrolló en el marco del proyecto macro de la creación del JuEGAS ProVEAS multifuncional.

\section{Etapa de documentación del modelo del alumno en JuEGAS_ProVEAS} MUTIFUNCIONAL: Aquí se documentó el modelado del alumno, de acuerdo a los diferentes ámbitos del Sistema multifuncional (ámbito evaluador, ámbito tutor, ámbito ejercitador).

Etapa del modelado y explicación: en esta etapa se diseñará el modelado del alumno con ayuda de las redes bayesianas y se perfeccionarán los roles, actividades, artefactos y tareas de la metodología SECMALI relacionadas con el modelado del alumno.

\section{Etapa del modelado y explicación}

Fase de elaboración parte II (SECMALI): para diseñar el modelado del alumno se debe seleccionar una estructura que represente dicho modelo; en este caso, selecciono la red bayesiana para representar el modelado del alumno dentro del JuEGAS_ProVEAS MULTIFUNCIONAL.

Según(GIRALDO,2006) las redes bayesianas son importantes porque se pueden utilizar en el modelado del alumno de múltiples formas, integrando suavemente inferencia diagnóstica y predictiva, con varias posibilidades de construir las redes dinámicamente. Una vez realizado el esfuerzo de especificación e implementación de las redes bayesianas podremos disfrutar de sus ventajas:

- Consistencia: el mal funcionamiento no es debido al mecanismo de inferencia.

- Explicaciones: el papel de las inferencias se describe a través de la naturaleza de las relaciones causales de las variables representadas en la red.

- Comunicación: utilizar una técnica ampliamente difundida, facilita la interacción (revisión, evaluación, comprensión, etc. del trabajo) con otros colegas.

Según (López, et al. 2007) la red bayesiana es importante porque presenta ventajas frente a los sistemas expertos clásicos basados en reglas cuando se van a utilizar, por ejemplo, para la toma de decisiones. En primer lugar, las redes bayesianas representan toda la información en un único formato (probabilístico y gráfico) lo que hace sencillas las interpretaciones. Además, permite retractarse de conclusiones obtenidas con anterioridad que ya no son razonables a la luz de nuevas evidencias, nos proporcionan una visión general del problema, generan un conjunto de alternativas ordenadas y facilitan la explicación de las conclusiones.

El tipo de Modelado del Alumno surge a partir del análisis de los tipos de modelado del alumno, abordados por (Millan, 2000). Para esta investigación se ha denominado "Modelo Unificado", sus características son las siguientes: 
Tabla 3. Tipo de Modelado del alumno para el JuEGAS_ProVEAS multifuncional

\begin{tabular}{|l|l|}
\hline \multicolumn{1}{|c|}{ Modelado del alumno } & \multicolumn{1}{|c|}{ Modelo unificado } \\
\hline conocimiento del alumno & $\begin{array}{l}\text { El conocimiento del alumno es una combinación entre su } \\
\text { conocimiento previo y el conocimiento del experto. }\end{array}$ \\
\hline interpretación & $\begin{array}{l}\text { Busca representar explícitamente tanto el conocimiento del alumno } \\
\text { como lo que le puede brindar el experto sobre la temática. }\end{array}$ \\
\hline Observaciones & $\begin{array}{l}\text { El modelo contempla la posibilidad al estudiante de interiorizar la } \\
\text { forma de adquisición de su propio conocimiento. }\end{array}$ \\
\hline
\end{tabular}

\section{Características particulares del modelo propuesto}

El modelamiento del estudiante en el JuEGAS_ProVEAS multifuncional se estructurara de la siguiente manera: cuando el estudiante ingresa por primera vez al JuEGAS debe crear su registro o en caso contrario, actualizar sus datos. Tiene dos posibilidades o dos modos de funcionamiento dentro del JuEGAS.

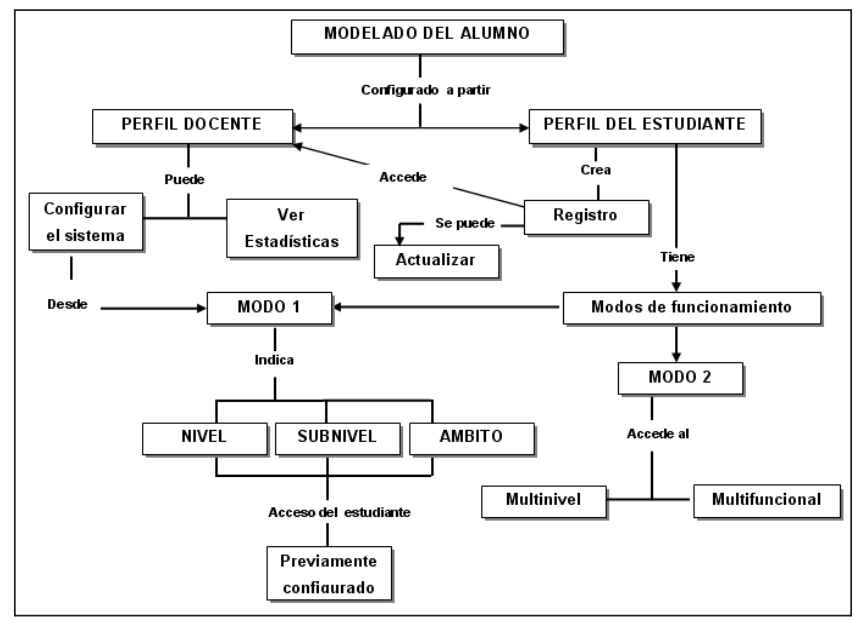

Figura 2. Características del modelo del alumno

Modo 1. Configurado por el docente: en este espacio el docente tiene la posibilidad de seleccionar el nivel, subnivel y ámbito según las necesidades educativas que él desee que sus estudiantes desarrollen. Si el estudiante ingresa al modo configurado por el docente solo puede acceder a lo previamente indicado por aquel. Por ejemplo, si el docente previamente escogió el ámbito tutor y dentro de éste ámbito seleccionó presentar al estudiante los problemas que se desarrollen dentro del nivel Cambio; el estudiante ingresará solo a ese nivel. Sin embargo, si el docente escoge un nivel de rango superior y por algún motivo el estudiante no es capaz de asimilar los conceptos de este nivel el sistema lo bajara de nivel. 
Modo 2. Ingreso independiente: desde este espacio el estudiante tiene acceso a todos los niveles (multinivel) y todos los ámbitos (multifuncional). En este caso ingresará, en primer instancia, al ámbito evaluador, diseñado para proponer ejercicios de ProVEAS con los cuales se busca valorar los resultados finales de los ejercicios y determinar las falencias del estudiante, una vez detectadas y analizadas estas falencias, con ayuda de la red bayesiana, se guiará al estudiante al ámbito tutor; éste ámbito se encarga de enseñar paso a paso las estrategias adecuadas para solucionar los ProVEAS, así como, los procesos seguidos para resolverlos.

Luego de ello, el estudiante pasará al módulo ejercitador donde podrá fortalecer sus conocimientos y una vez termine de hacerlo, el sistema lo enviará directamente al ámbito evaluador donde verificará si realmente aprendió y le dará la opción de continuar o salir completamente del JuEGAS_ProVEAS multifuncional.

\section{Definición de las variables para el modelamiento del estudiante}

Se trabajará con base en dos variables; la primera tendrá en cuenta la información obtenida del estudiante a medida que realiza la interacción con el JuEGAS_ProVEAS multifuncional y la segunda, corresponde a los aspectos funcionales del JuEGAS_ProVEAS multifuncional.

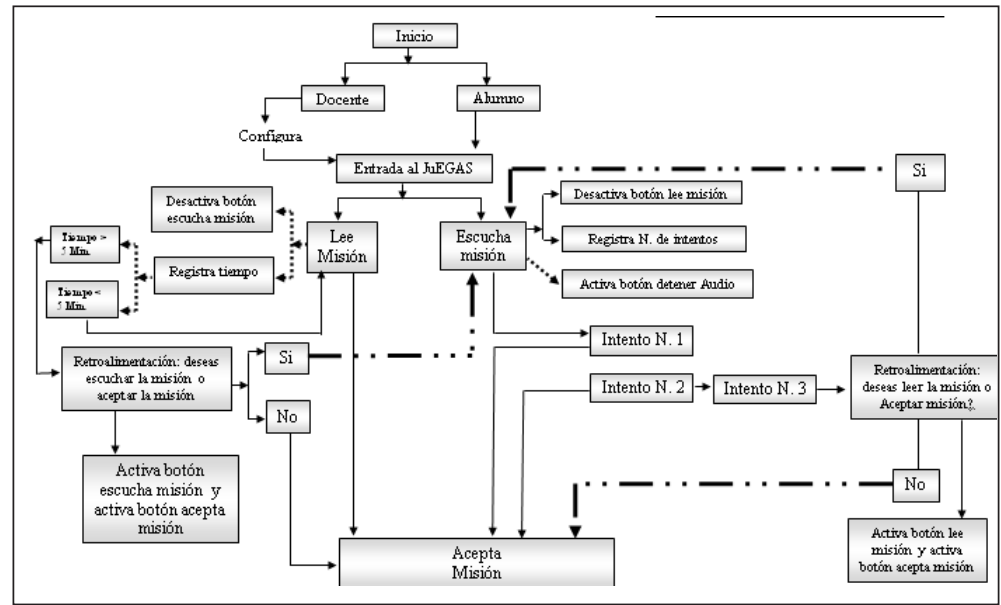

Gráfica 1. Entrada al JuEGAS_ProVEAS MULTIFUNCIONAL, adaptado de (Castillo, 2008)

A. Información obtenida del estudiante: cuando el usuario ingresa por primera vez al JuEGAS_ProVEAS MULTIFUNCIONAL debe escribir sus datos personales para registrarse, los cuales son usados en el proceso del modelado del alumno. Dicha información contiene el nombre, apellidos, edad y código del usuario con los cuales se hace un control de su ingreso, un seguimiento de los aciertos, los errores y de las estrategias a las que accede cuando resuelve los ejercicios. Además, se guarda un registro de las pantallas visitadas, el tiempo utilizado en la solución de los ejercicios de ProVEAS entre los diferentes ámbitos por estudiante y por ejercicio dentro del JuEGAS_ProVEAS MULTIFUNCIONAL. 


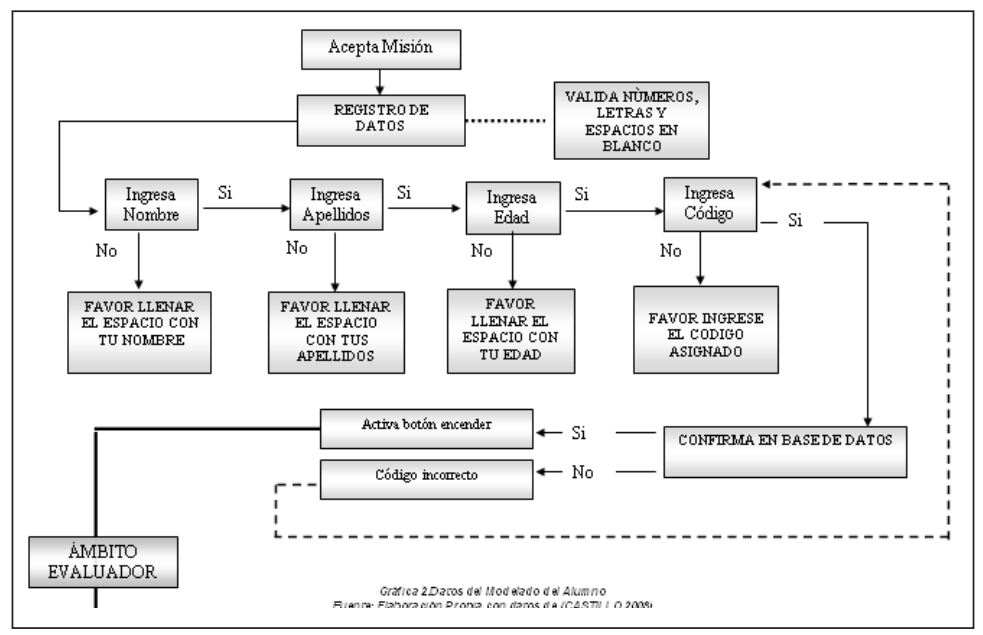

Gráfica 2. Datos del Modelado del Alumno, adaptado de (Castillo, 2008)

B. Aspectos funcionales del JuEGAS_ProVEAS multifuncional: luego del registro del estudiante, el sistema multifuncional genera, en forma aleatoria y automática, los ejercicios para que pueda solucionarlos dependiendo del módulo con el cual esté interactuando.

Si el estudiante ingresa por el Modo Independiente, inicia en el ámbito evaluador, donde el sistema hace un diagnóstico de los conocimientos previos del alumno, con la finalidad de evaluar el dominio que tiene el estudiante sobre el sistema de conocimiento que se implementa en el JuEGAS_ProVEAS MULTIFUNCIONAL.

Con este diagnóstico, el sistema enviará al estudiante al ámbito tutor donde se le enseñarán las estrategias para solucionar ejercicios de ProVEAS, en este ámbito se realizarán preguntas control para verificar si el estudiante ha asimilado el proceso e ingresa al ámbito ejercitador donde se le plantean tres ejercicios de ProVEAS por cada subnivel.

En este orden de ideas, el sistema le envía un mensaje preguntando al estudiante si desea salir del sistema o evaluar nuevamente sus conocimientos. En caso de escoger ser evaluado, accede al ámbito evaluador para hacer una valoración final que permitirá establecer si ha habido una mejora en la solución de los ejercicios. Cabe resaltar que si el estudiante escoge la opción salir, entonces se premia por su desempeño en el juego con un diploma de reconocimiento. Además, luego de pasar por el evaluador nuevamente también obtiene el diploma. En el análisis de los datos se tiene en cuenta todo el proceso de los diferentes ejercicios resueltos por el estudiante. 


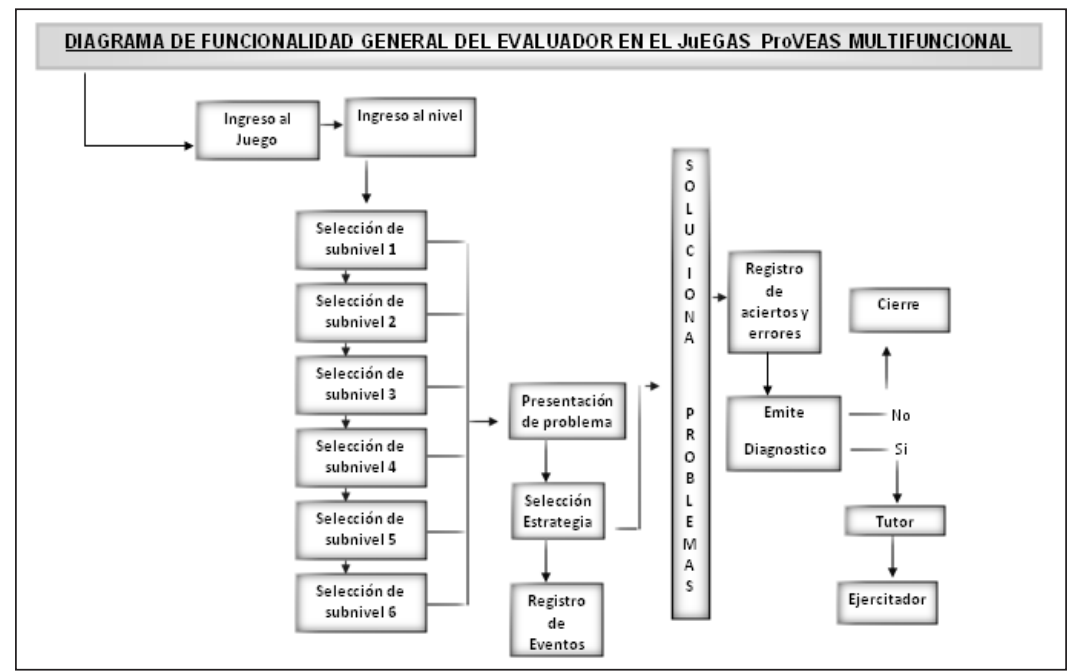

Diagrama 1. Evaluador JJuEGAS_ProvEAS MULTIFUNCIONAL

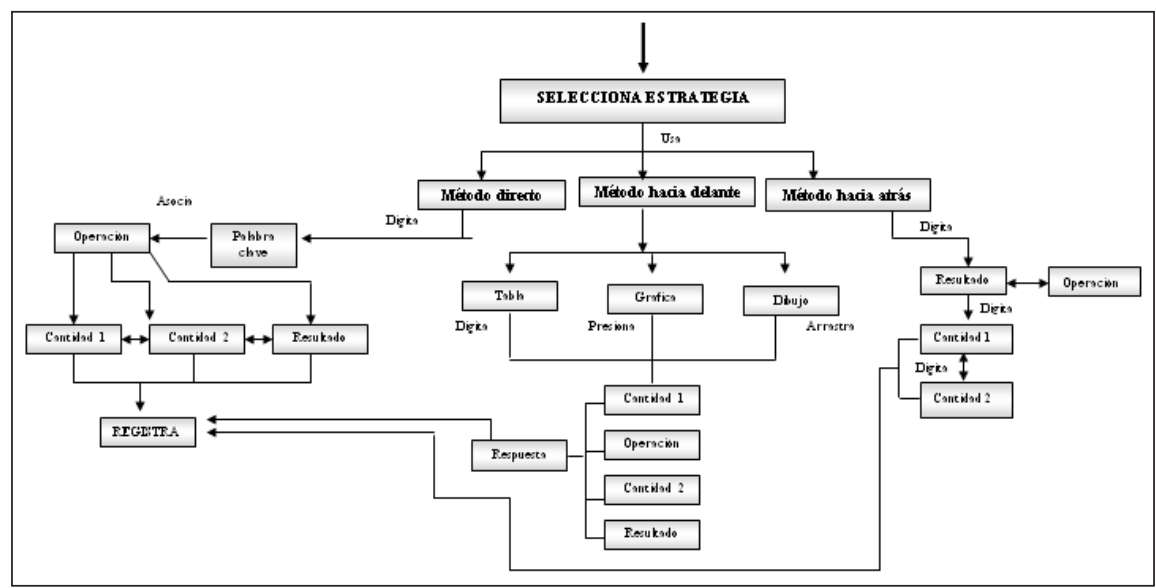

Figura 3. Selección de estrategia en el ámbito Evaluador modelado del alumno JuEGAS_ProVEAS MULTIFUNCIONAL, adaptado de (Castillo, 2008) 


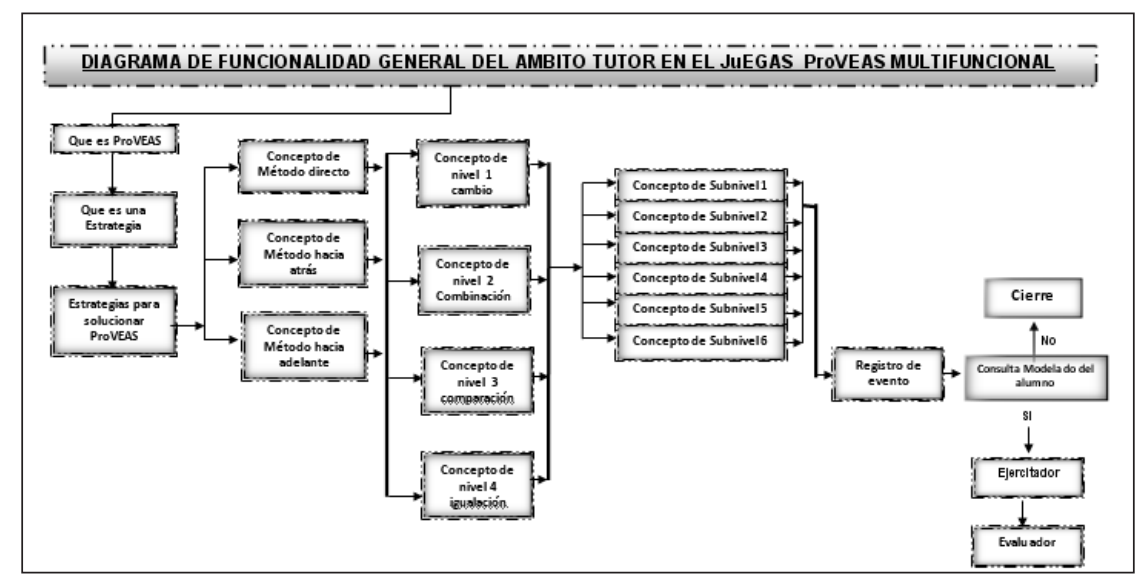

Diagrama 2 . Ámbito Tutor JuEGAS_ProVEAS MULTIFUNCIONAL

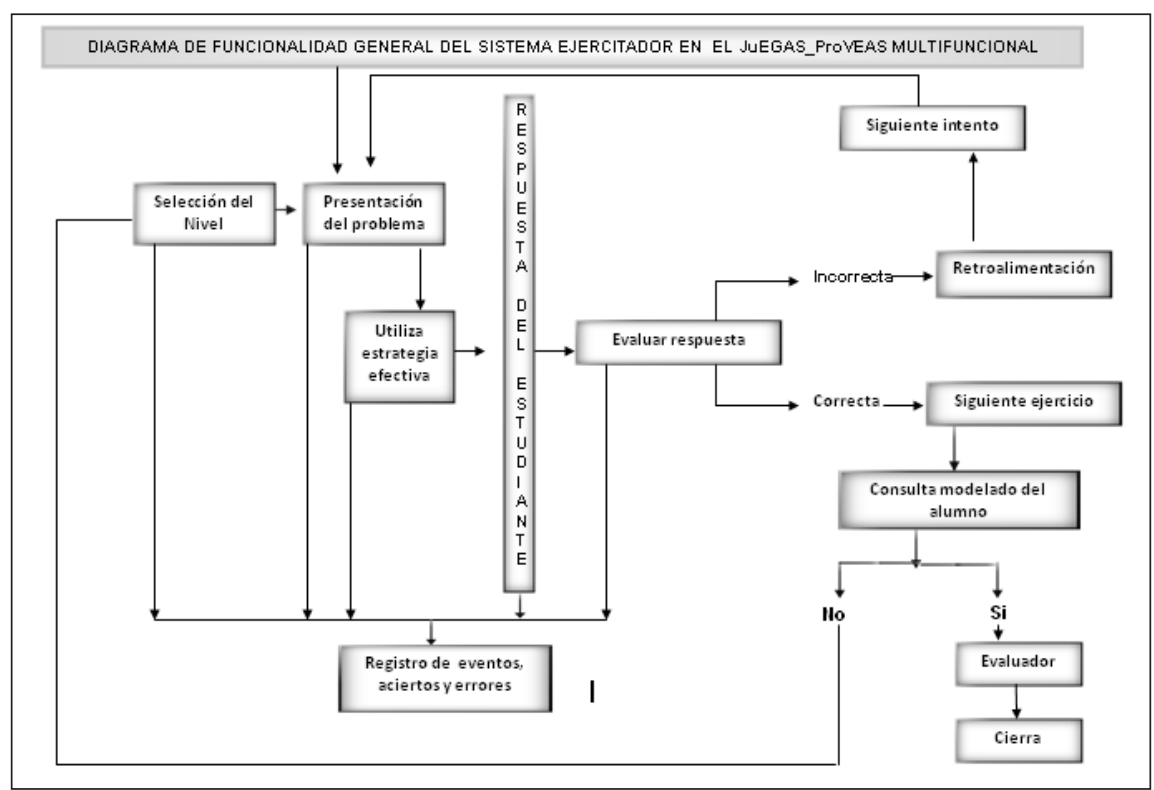

Diagrama 3. Ejercitador JuEGAS_ProVEAS MULTIFUNCIONAL 


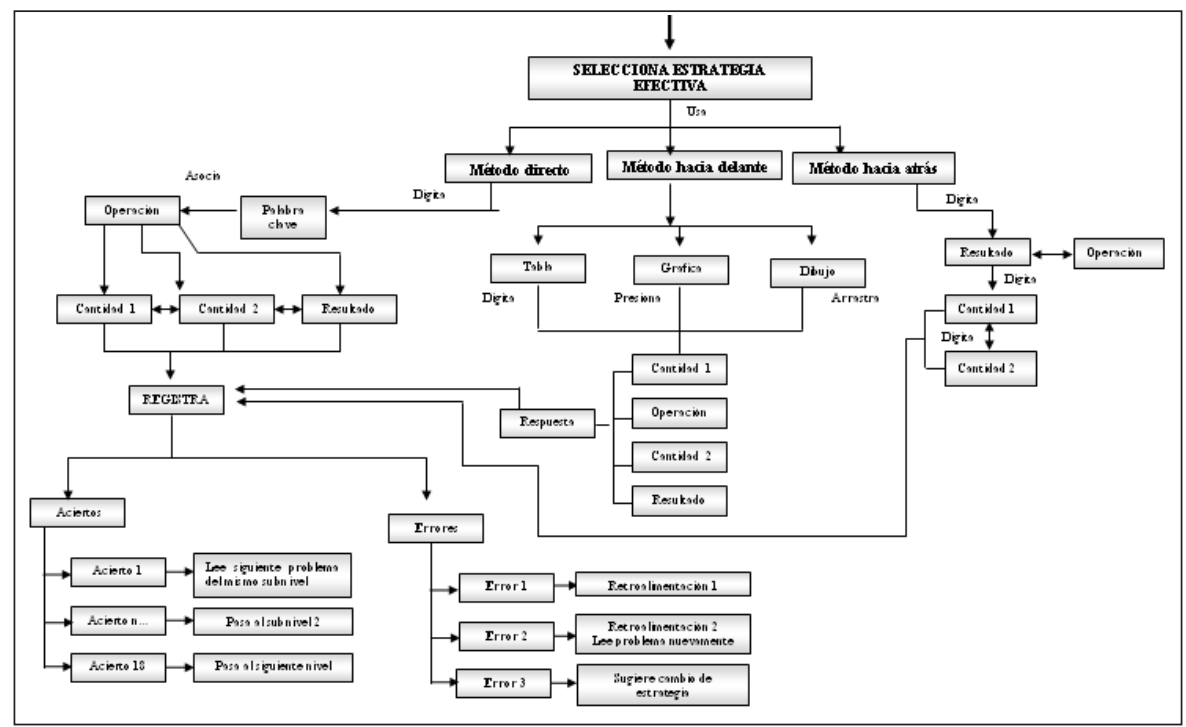

Figura 4. Selección de la estrategia efectiva en el ámbito ejercitador (JuEGAS_ProVEAS

MULTIFUNCIONAL), adaptado de (Castillo, 2008)

\section{Definición de los criterios para estructurar el modelado del alumno en SECMALI}

Para realizar el modelado del alumno dentro de un JuEGAS, hay que tener claridad en las siguientes actividades, tareas, roles y artefactos del la metodología SECMALI.

Modelo de contenido: la actividad del modelo de contenidos es desarrollada por el rol del pedagogo dentro de la metodología SECMALI. En este sentido, la persona que adopta el rol del Modelador del Sistema Tutor Inteligente debe trabajar en conjunto con el pedagogo para hacer eficiente la creación del modelado del alumno.

Es de vital importancia realizar un análisis detallado de los temas que se desea enseñar en el software; en este caso específico, se realizó un análisis detallado de los ProVEAS, los niveles y subniveles de complejidad y de esta manera hizo claridad sobre lo que se pretende enseñar al estudiante, con el fin de representar computacionalmente la estructura conceptual del alumno frente a la temática del JuEGAS.

Modelo del experto: el modelo del experto contiene todas las posibilidades que el estudiante puede realizar en el JuEGAS, en otras palabras es la representación del conocimiento de los temas tratados en el juego, pero en el contexto de las actividades del juego. A su vez recoge las interacciones definidas en la Mecánica del Juego y modela sus relaciones; esta información se convierte en los objetivos, las creencias, los planes y los comportamientos de los agentes del sistema. 
El agente que hace las veces de tutor, deberá tener la representación total del sistema y tener acceso a las decisiones que asume cada personaje en particular. De esa manera, compara las acciones que un agente autónomo haría con las que hace el estudiante y brinda las ayudas necesarias a los estudiantes cuando estos las requieran.

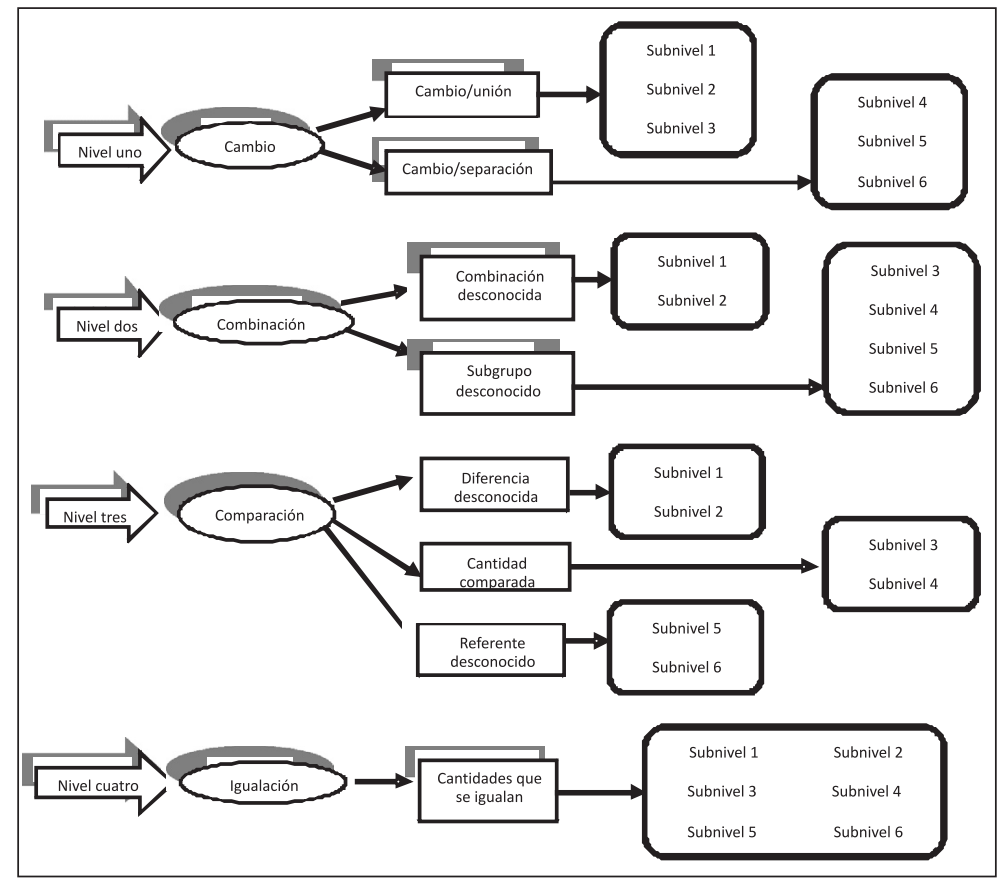

Figura 5. Artefacto: Sistema de conocimiento del JJuEGAS_ProvEAS MULTIFUNCIONAL, adaptado de (Castillo, 2008)

Modelo de interfaz: el modelo de interfaz es un elemento fundamental en el modelado del alumno porque este es el módulo que tiene el rol del tutor propiamente dicho a través de representaciones graficas. Es el que lleva a la práctica los objetivos del módulo pedagógico y controla la secuenciación de los contenidos. Además, es el que permite la comunicación con otros estudiantes o usuarios; la comunicación con el tutor, el cual debe ser un personaje de la historia; la información general del micromundo para apoyar la toma de decisiones. La zona de herramientas y acciones del rol que está desempeñando el estudiante.

Modelo pedagógico: en la metodología SECMALI, la persona que adopta el rol del pedagogo se encarga de definir el modelo pedagógico del proyecto, resaltando las características de trabajo colaborativo. Debe elegir las teorías de aprendizaje con mayor adaptación a la propuesta educativa y las características del juego.

En este modelo se indica la usabilidad, navegabilidad del software, el objeto de conocimiento, el ambiente de aprendizaje, la evaluación, la ayuda y los lineamientos que orientarán la toma 
de decisiones en el JuEGAS; estas características del Modelo Pedagógico son esenciales en la modelación del alumno.

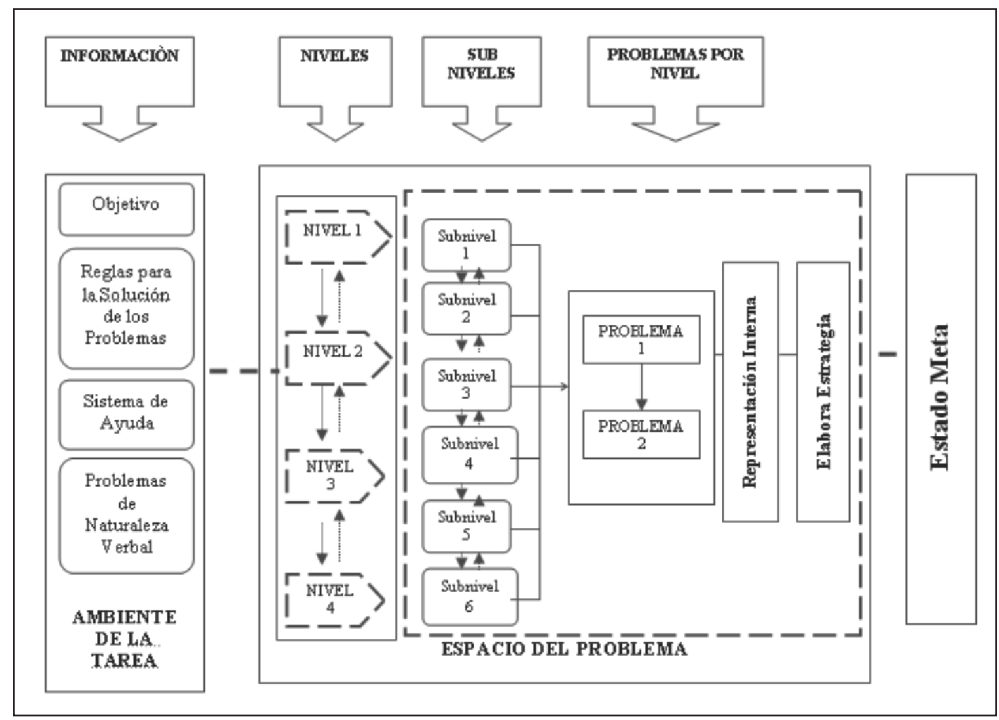

Figura 6. Modelo Pedagógico, tomado de (CASTILLO, 2008)

En el caso del JuEGAS_ProVEAS MULTIFUNCIONAL se diseñó un modelo pedagógico constructivista tomando como referente teórico por un lado, la investigación (Riley, Greene Y Heller, 1983) según la estructura semántica, el ambiente de la tarea y el espacio del problema los cuales se convierten en aspectos básicos del modelo pedagógico del JuEGAS. Y por el otro lado, lo que plantean (Newel y Simón, 1972), sobre el ambiente de la tarea.

A partir del modelo pedagógico de (Castillo, 2008) se configura la estrategia para dar estructura al sistema multifuncional. Este es un modelo pedagógico basado en (Riley, Greeno y Heller, 1983), con elaboración propia para el sistema multifuncional.

Tabla 4. Estrategia para la estructuración del JuEGAS_ProVEAS multifuncional

\begin{tabular}{|c|c|c|c|c|}
\hline & EVALUADOR & TUTOR & EJERCITADOR & MULTIFUNCIONAL \\
\hline Nivel & * & * & * & 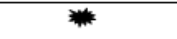 \\
\hline -.......... & * & 业 & \% & * \\
\hline$\rightarrow$ Contenidos & & 牶 & & 牶 \\
\hline$\rightarrow$ Prea. Control & & 粦 & & * \\
\hline Problema & \# & & * & 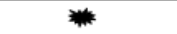 \\
\hline$\rightarrow$ Retroalimenta & & & 絭 & * \\
\hline$\longrightarrow$ Calificación & \% & & & w \\
\hline L.... Subnivel & & & & \\
\hline
\end{tabular}


En la estrategia para estructurar el modelado del alumno se tienen en cuenta las características particulares de cada ámbito a la hora de presentar la información y el contenido del JuEGAS a los usuarios. La presentación de la información se hace como se presenta a continuación.

En el ámbito evaluador tiene acceso a los niveles, subniveles, problemas planteados y la calificación obtenida, puesto que la finalidad del evaluador es realizar un diagnóstico del conocimiento y el proceso llevado a cabo por los estudiantes cuando solucionan los ProVEAS. En el espacio tutor se podrá tener acceso a los niveles, subniveles, contenidos y preguntas de control para verificar la asimilación del contenido, puesto que el tutor tiene la finalidad de enseñar a los estudiantes a solucionar de forma adecuada los ProVEAS . En el campo ejercitador se tiene acceso a los niveles y subniveles, como también a los problemas y las retroalimentaciones. En el ejercitador se pretende entrenar a los estudiantes en la forma efectiva de solucionar cualquier ProVEAS . Y por último, el sistema multifuncional configura todas las posibilidades de interactuar con el JuEGAS_ProVEAS multifuncional. Con él se busca que el sistema evalué el dominio de conocimiento del estudiante, genere un diagnóstico acerca del dominio de los ProVEAS y, con base en él, conduzca al estudiante al ámbito tutor, en el cual se le enseña paso a paso los ProVEAS y la estrategia eficaz para solucionarlos. Por último, el estudiante ingresa al ámbito ejercitador para fortalecer su aprendizaje y dominar la estrategia eficaz en la solución de ProVEAS .

Las estrategias de aprendizaje: las estrategias utilizadas hacen parte del modelado del alumno, ya que estas estrategias permiten identificar qué método está utilizando el estudiante para resolver el problema y en conjunto con el modelo del experto permite identificar la posibilidad de éxito que puede tener el estudiante cuando soluciona un problema. Cabe resaltar que las estrategias de aprendizaje nacen del modelo pedagógico y en el caso JuEGAS ProVEAS multifuncional se hace énfasis en las estrategias de método hacia delante, método hacia atrás y método directo utilizadas en la solución de los ProVEAS.

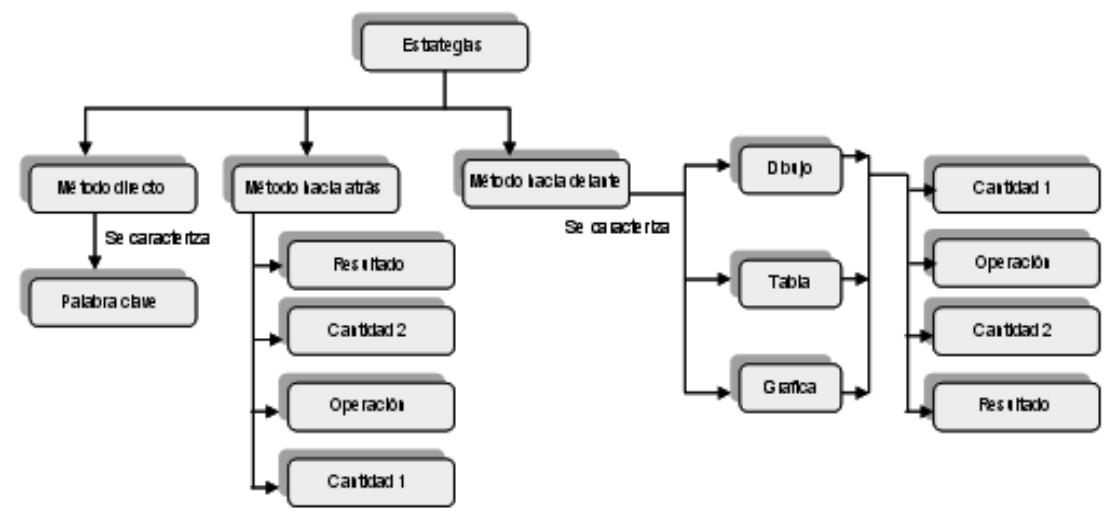

Figura 7. Estrategias usadas por los sujetos en la solución de PNV 
Estructura para representar el modelado del alumno: existen varias estructuras que permiten representar la estructura del modelado del alumno. Entre ellas tenemos: sistemas basados en reglas, lógica difusa, red bayesiana, entre otras. (Millán, 2000), afirma que la estructura adecuada, para llevar a cabo el modelado del alumno es a través de una red bayesiana, porque ofrece una base teórica para definir dicho modelo.

Además, la arquitectura que se establece presenta una forma más efectiva de comunicación entre el usuario y el sistema. Por otro lado, (Giraldo, 2006) afirma la utilización de la red bayesiana como la estructura que permite representar el modelado del alumno en el JuEGAS. Por su parte (Ramírez, 2005), implementa la red bayesiana en un sistema ejercitador y reafirma las siguientes características sobre dicha red: la red bayesiana sirve de base para la toma de decisiones en el complejo proceso de definición del modelamiento del estudiante.

Las redes bayesianas permiten hacer predicciones de los resultados que se desea obtener en el sistema sobre el estudiante. “...Este razonamiento probabilístico, es especialmente adecuado para tratar con un mundo variable, que más que a conclusiones inequívocamente ciertas nos lleva a conclusiones probablemente ciertas" (Llorens, 2000). Integran la inferencia diagnóstica y predictiva, con varias posibilidades de construir las redes dinámicamente en la definición del modelado del alumno.

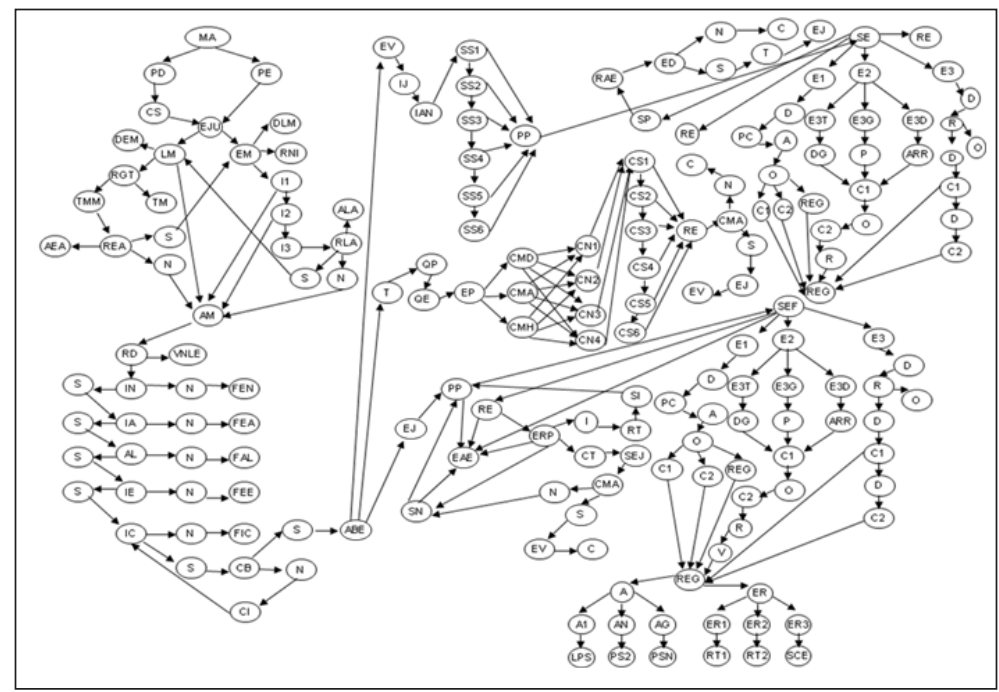

Diagrama 4. Red Bayesiana del modelado del alumno

La Red bayesiana es la técnica más utilizada y exige iniciar con una probabilidad a priori. Dicha red es un conjunto de variables, una estructura gráfica que conecta estas variables y un conjunto de distribuciones de probabilidad condicional. La descripción más frecuente de una red bayesiana se basa en dos elementos: una dimensión cualitativa y otra cuantitativa. Podemos construir un modelo gráfico probabilístico a partir de una base de datos que contenga un conjunto de observaciones sobre un conjunto de variables. 
Datos personales: para identificar y crear en el sistema, el modelado del alumno es necesario que cada estudiante ingrese sus datos personales como: el nombre, la edad, el alias o nick, entre otros datos, de acuerdo con la pertinencia de los creadores del JuEGAS. Estos datos son muy importantes y permiten la realización del historial del alumno.

Historial de registro: cuando el estudiante empieza a interactuar con el sistema multifuncional se requiere hacer un registro de aciertos y errores, que van a posibilitar la creación del historial del alumno o historial de registro, es por eso que cada cosa que el estudiante realice dentro del software se registra en una base de datos. A la hora de caracterizar el modelado del alumno, se toman estos datos representativos que corresponden al modelado del alumno individual dentro del JuEGAS_ProVEAS MULTIFUNCIONAL.

El historial de registro es el que va guardando todos los aciertos, errores, registros del estudiante y se va actualizando cada vez que este ingrese. Es muy importante tener un historial de registro porque es el que indica si el estudiante ha aprendido o no. Como también permiten la actualizando del modelado del alumno.

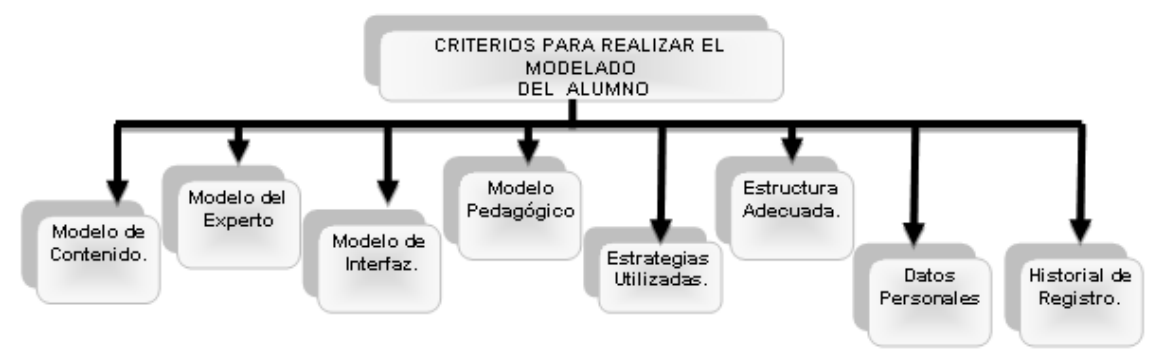

Figura 8. Criterios para elaborar el modelado del alumno dentro del JuEGAS_ProVEAS MULTIFUNCIONAL

\section{CONCLUSIONES}

\section{Desde el punto de vista pedagógico}

El modelado del alumno busca representar el pensamiento del estudiante cuando está frente a la solución de un problema en general. En otras palabras, son los procesos lógicos y mentales que un estudiante realiza internamente y le brindan la posibilidad de llegar a la solución de un problema planteado.

(Millán, 2000) explica los modelados del alumno, más utilizados. Sin embargo, ninguno de estos se adapta a las características propias del Sistema MULTIFUNCIONAL. Por lo tanto, fue necesario definir un modelo nuevo adaptado a los JuEGAS, dicho modelo se denomina "Modelado unificado" y presenta las siguientes características: 
Una vez identificado el tipo de modelo que se va a trabajar es necesario seleccionar la estructura para representar dicho modelo. En este caso, teniendo en cuenta las características de los JuEGAS, se propone adoptar la red bayesiana.

El sistema multifuncional se convierte en una herramienta didáctica y pedagógica para el docente, puesto que permite combinar tres criterios académicos como lo son: evaluar, tutorizar y ejercitar a los estudiantes mientras adquieren un conocimiento nuevo. En este caso, identifican y utilizan las estrategias para resolver problemas de naturaleza verbal de estructura aditiva y sustractiva.

\section{Desde el punto de vista tecnológico}

Para definir los criterios del modelado del alumno en Juegos Educativos Gestionados con Agentes Software (JuEGAS) MULTIFUNCIONALES, fue necesario vivir paso a paso la especificación SECMALI, comenzando por identificar los roles y cada una de las fases de SECMALI. El proceso se dio en forma iterativa y colaborativa, puesto que cada rol dentro de la metodología SECMALI guarda una relación directa con los demás.

Los criterios definidos para realizar el modelo del alumno dentro del JuEGAS_ProVEAS MULTIFUNCIONAL, son los siguientes:

Sistema multifuncional: este concepto surge de la investigación y se define como un sistema desarrollado con el fin de implementar 3 ámbitos pedagógicos: evaluar, ejercitar y tutorizar las estrategias de aprendizaje de los estudiantes.

Después de revisar diferentes autores y bibliografías, se concluyó que el sistema MULTIFUNCIONAL, es innovador en su diseño y creación. Con base en esta idea se puede afirmar que es la primera vez que se realiza un sistema que permite evaluar, ejercitar y tutorizar las estrategias de aprendizaje de los estudiantes, implementadas a través de tres componentes (evaluador, tutor y ejercitador) separados e interrelacionados teniendo en cuenta las necesidades de aprendizaje del usuario.

Este software tendrá la posibilidad de ser configurado por el docente, ya que posee dos tipos de usuario (docente y alumno) para ingresar al juego. Cuando ingresa el docente, se brinda la posibilidad de que él pueda seleccionar el ámbito en el cual quiera trabajar, como también los niveles y los subniveles que él desee dar a conocer a sus estudiantes y así el sistema multifuncional estaría habilitado para el estudiante.

Cuando el usuario es el estudiante, se le presenta el sistema MULTIFUNCIONAL de acuerdo con la configuración especificada por el docente. Si el sistema no se ha configurado por el docentes o este lo configuró en el modo MULTIFUNCIONAL, se presentarán al estudiante todas las posibilidades del sistema, es decir, el ámbito evaluador, el ámbito tutor, y el ámbito ejercitador. 
Para solucionar problemas de naturaleza verbal de estructura aditiva y sustractiva (ProVEAS), este tipo de problemas implican hacer una representación mental donde se utilizan las operaciones matemáticos suma y resta para resolver ecuaciones aritméticas con una incógnita. Ejemplo: Juan tiene tres manzanas y María le da dos más. ¿Cuántas manzanas tiene Juan en total? Riley, Greene y Heller, identifican cuatro niveles que se utilizan en el planteamiento de los ProVEAS diferenciados por la ubicación de los conectores lógicos en la formulación del problema.

\section{Desde el punto de vista investigativo}

Esta investigación aporta elementos esenciales al macro proyecto JuEGAS_ProVEAS MULTIFUNCIONAL en cuanto a la definición del modelo pedagógico a implementar en el JuEGAS. Él ofrece el diseño de contenido de los ProVEAS, se detallan las estrategias seleccionadas en la solución de los ProVEAS para dicho software, se describe la configuración de cada uno de los ámbitos que conforman el sistema MULTIFUNCIONAL y las características principales de cada uno de ellos, se brinda ayuda en la definición de las características de la interfaz y la construcción de la historia del juego.

Estos elementos tienen un grado investigativo bastante avanzado, sin embargo, son susceptibles de ser mejorados a la hora de formalizar el proyecto macro y su implementación.

Además, la investigación: "Modelado del alumno en juegos educativos gestionados con agentes software (juegas) multifuncionales, el caso juegas_proveas multifuncional", se socializó por parte de la unidad investigativa en varios eventos de carácter local, departamental, nacional e internacional. Estas presentaciones fueron de vital importancia, los diferentes evaluadores resaltaron la importancia y pertinencia del proyecto; las recomendaciones hechas en cada uno de los eventos fueron significativas para mejorar y documentar la investigación. Ocupó el tercer puesto en el $10^{\circ}$ premio de Informática Educativa, categoría investigación, organizado por la Red Iberoamericana de Informática Educativa Nodo Colombia (Ribie-Col).

\section{REFERENCIAS BIBLIOGRÁFICAS}

BARCHINI Graciela Elisa. (2005). Métodos “I + D” de la Informática Revista de Informática Educativa y Medios Audiovisuales Vol. 2(5), págs. 16-24. Universidad Nacional de Santiago del Estero. Argentina. Consultado el 15 Noviembre de 2008.

BEGOÑA Groos, (1996). Diseño y evaluación de software educativo. Editorial Ediciones graficas herrera y asociados, Bogota. Colombia. Consultado el 15 Septiembre de 2008.

CARRETERO DÍAZ Antonio María. (2001). Metodología didáctica para enseñanza de Geometría Descriptiva basada en un Tutor-Evaluador y en un Generador de ejercicios integrados en un entorno de propósito constructivo general. Universidad Politécnica de Madrid. Consultado el 17 de Julio de 2008. 
CARRO, R.M., BREDA, A.M., CASTILlO, G., BAJUELOS, A.L. (2002). Generación de Juegos Educativos Adaptativos. En: Interacción 2002. Actas del III Congreso Internacional de Interacción Persona-Ordenador, Eds. Aedo, I., Cuevas, P. and Fernández, C. (Granada: AIPO), 164-171.

CASTILlO, Mónica. (2008). Estrategias Usadas por expertos y novatos en la solución de Problemas de Naturaleza Verbal de Estructura Aditiva y Sustractiva. Universidad Pedagógica Nacional. Bogota.

CATALDI, ZULMA. SALGUIERO, FERNANDO Y LAGE, FERNANDO. "Sistemas Tutoriales Inteligentes con Modelado del Estudiante y del autor" Edutec. Revista Electrónica de Tecnología Educativa. Núm20/enero2006. consultado en htt:/edutec. rediris.es/Revelec2/revelec20/zulma20.pdf. el 9 de marzo de 2008.

GIRALDO, CARDOZO JUAN CARLOS. (2007). Maestria en Ingenieria de Sistemas y Computación, Universidad del Valle. "Modelado de alumno en software Educativo, Distribuido, Multimedia, Adaptativo, Lúdico e interactivo" consultado el 11 de marzo 2008.

GONZÁLEZ, SOLEDAD. (2004). Sistemas Inteligentes en la Educación: una revisión de las líneas de investigación y aplicaciones actuales. RELIEVE 10(1):3-22. España. Consultado el 7de marzo 2008.

HEGARTY, M., MAYER, R. E., Y MONK, C. A. (1995). Comprehension of arithmetic word problems: a comparison of successful and unsuccessful problems solvers, Journal of Educational Psychology, 87,18-32. Disponible en http://psycnet.apa.org/index. cfm?fa=buy.option ToBuy\&id=1995-20055-001.

HERNANDEZ SAMPIERI, ROBERTO; FERNÁNDEZ COLLADO, CARLOS; BAPTISTA LUCIO, PILAR (1997). Metodología de la Investigación. Mc Graw Hill, México. Consultado el 15 febrero de 2009.

LÓPEZ Puga, Jorge. GARCÍA García, Juan. DE LA FUENTE Sánchez, Leticia y DE LA FUENTE Solana, Emilia Inmaculada. (2007). Las redes bayesianas como herramientas de modelado en psicología. Edición web www.um.es/analesps): 1695-2294. Universidad de Murcia. Murcia (España).

MILLANV.EVA. “Sistema Bayesiano para el modelado del alumno”. Tesis doctoral, Departamento de Lenguajes y Ciencias de la Computación, Universidad de Málaga. España. 2000 consultado en htt://www.Icc.uma.es/eva/investigación/SBMA.pdf. el 1 de Marzo de 2008.

MORENO S., Mario. R. (2002). Modelado del Estudiante para un Ambiente de Aprendizaje de Lecto/Escritura. Tesis Maestría. Ciencias con Especialidad en Ingeniería en Sistemas Computacionales. Departamento de Ingeniería en Sistemas Computacionales, Escuela de Ingeniería, Universidad de las Américas Puebla. Mayo. 